\title{
Multilevel Logistic Regression Analysis Applied to Binary Contraceptive Prevalence Data
}

\author{
Md. Hasinur Rahaman Khan and J. Ewart H. Shaw \\ University of Warwick
}

\begin{abstract}
In public health, demography and sociology, large-scale surveys often follow a hierarchical data structure as the surveys are based on multistage stratified cluster sampling. The appropriate approach to analyzing such survey data is therefore based on nested sources of variability which come from different levels of the hierarchy. When the variance of the residual errors is correlated between individual observations as a result of these nested structures, traditional logistic regression is inappropriate. We use the 2004 Bangladesh Demographic and Health Survey (BDHS) contraceptive binary data which is a multistage stratified cluster dataset. This dataset is used to exemplify all aspects of working with multilevel logistic regression models, including model conceptualization, model description, understanding of the structure of required multilevel data, estimation of the model via the statistical package $M L w i N$, comparison between different estimations, and investigation of the selected determinants of contraceptive use.
\end{abstract}

Key words: BDHS, CPR, cluster, division, MCMC, MLwiN, multilevel, MQL, PQL, TFR.

\section{Introduction}

Bangladesh is the most densely populated country in the world. The country has currently a population about 150 million, with a corresponding population density of 939 per square kilometer and growth rate of $1.42 \%$ (M. Anwarul Iqbal, 2008). In the second half of the last century, the population grew extraordinarily rapidly, tripling during the period, whereas during the entire first half of the century the population increased by only $45 \%$. Family planning was introduced in Bangladesh in the early 1950s. The policy to reduce fertility rates has been repeatedly reaffirmed by the Government of Bangladesh since liberation in 1971. During the mid 1970s, the contraceptive prevalence rate (CPR) was less than $10 \%$ and the total fertility rate (TFR) was more than 6 births per women (Islam and Islam, 1993). The subsequent last two rounds of the BDHS, in 1999-2000 
and 2004, found CPRs of 54\% and 58\%, respectively, the TFRs for those years were 3.3 and 3.0 (NIPORT, 1999-2000, 2005).

The association between estimated levels of contraceptive prevalence and the level of fertility is very close in the higher fertility countries like Bangladesh (Curtis and Diamond, 1995). As per the widely accepted correlation between CPR and TFR, a rise of $9 \%$ points in CPR has been seen to be accompanied by a fall of 0.64 in the TFR (Mauldin and Segal, 1988). Bangladesh still lacks such an estimate, which raises questions about the country's fertility and contraceptive dynamics and prospects for future fertility decline. Use of contraception is the main contributor of fertility declining, as has been shown by many research workers (Cleland et al., 1994; R. Amin et al., 1994; Rani and Radheshyam, 2007).

Fertility decline should continue if the wider use of contraception continues in all levels and groups of people in Bangladesh. It is critical for family planning workers to continue to meet the needs of existing family planning users, and also to address unmet need for family planning since individual tastes, interests, behaviours, etc. differ from one unit to another within each level, owing to variability among various socioeconomic and geographical factors such as religion, culture, income, place of residence, education, occupation, mass media access, administrative and social facilities, and so on. That is why their efforts and approaches do not seem to be equally effective, evenly served or acknowledged in some areas. As a result, the effectiveness of the program varies considerably. It is necessary to assess the within- and between-level variation, and to estimate the true effect of the above-mentioned factors on CPR, in order to implement more effective future family planning policies that target particular units at various levels of the hierarchy.

This paper highlights the importance of multilevel analysis using logistic regression models for studying contraceptive prevalence in Bangladesh from the multistage clustered 2004 BDHS data. The paper aims to investigate the selected factors affecting the regulation of fertility through contraception in the context of multilevel modeling. It also aims to measure the influence of the combination of the selected factors on the current contraceptive practice of women in Bangladesh, and emphasis is given to exploring the true effect of the factors on the contraceptive prevalence taking into consideration the effect of the levels. The analysis is mainly carried out using MLwiN (Rasbash et al., 2004).

\section{The Multilevel Model}

\subsection{Multilevel analysis for multistage clustered data}

In multilevel research, the structure of data in the population is hierarchical, and a sample from such a population can be viewed as a multistage sample. 
Because of cost, time and efficiency considerations, stratified multistage samples are the norm for sociological and demographic surveys. For such samples the clustering of the data is, in the phase of data analysis and data reporting, a nuisance which should be taken into consideration. However, these samples, while efficient for estimation of the descriptive population quantities, pose many challenges for model-based statistical inference.

This clustering sampling scheme often introduces multilevel dependency or correlation among the observations that can have implications for model parameter estimates. For multistage clustered samples, the dependence among observations often comes from several levels of the hierarchy. The problem of dependencies between individual observations also occurs in survey research, where the sample is not taken randomly but cluster sampling from geographical areas is used instead. In this case, the use of single-level statistical models is no longer valid and reasonable. Hence, in order to draw appropriate inferences and conclusions from multistage stratified clustered survey data we may require tricky and complicated modeling techniques like multilevel modeling, and very often the computation required for this is not straightforward and is not very time consuming as currently there is a number of software packages.

The 2004 BDHS data set used for this study is based on multistage stratified cluster sampling. The appropriate approach to analyzing contraceptive data from this survey is therefore based on nested sources of variability. Here the units at lower level (level-1) are individuals (ever-married women aged 10-49) who are nested within units at higher level (clusters: level-2) and the clusters are again nested within units at the next higher level (divisions: level-3). Clusters are primary sampling units (PSU) defined by the National Census of 1981, and correspond approximately to village in rural areas. All clusters are approximately of equal size in terms of area. On the other hand Divisions are administrative areas each of which consists of a number of sub-administrative areas called Zila. Due to this nested structure, the odds of women experiencing the outcome of interest are not independent, because women from the same cluster may share common exposure to community characteristics. The response variable in this study is "currently using contraception" which is binary and hence multilevel logistic regression model is a natural choice for modeling. Traditional logistic regression (which, in multilevel analysis terms, is single-level) requires the assumptions: (a) independence of the observations conditional on the explanatory variables and (b) uncorrelated residual errors. These assumptions are not always met when analyzing nested data. But the multilevel logistic regression analysis consider the variations due to hierarchy structure in the data. It allows the simultaneous examination of the effects of group level (cluster and division) and individual level variables on individual level outcomes while accounting for the 
non-independence of observations within groups. Also this analysis allows the examination of both between group and within group variability as well as how group level and individual level variables are related to variability at both levels.

\subsection{Parameter estimation}

The most common methods for estimating multilevel logistic models, used in this study, are based on likelihood. Among the methods, Marginal Quasi Likelihood (MQL) (Goldstein, 1991; Goldstein and Rasbash, 1996) and Penalized Quasi Likelihood (PQL) (Laird, 1978; Breslow and Clayton, 1993) are the two prevailing approximation procedures. After applying these quasi likelihood methods, the model is then estimated using iterative generalised least squares (IGLS) or reweighted IGLS (RIGLS) (Goldstein, 2003). Second-order PQL method has been used throughout the multi-level analyses since this method approximates well compared to the other PQL and MQL methods (Goldstein, 2003). Details of the PQL method are given below. Bayesian methods using Markov chain Monte Carlo (MCMC) have also been used for parameter estimation.

\section{Penalized quasi-likelihood}

The PQL estimation procedure is described here for two level logistic regression models. Consider a level-1 outcome $Y_{i j}$ taking on a value of 1 with conditional probability $p_{i j}$. Then the logit model or the generalized linear model is,

$$
\ln \left[\frac{p_{i j}}{1-p_{i j}}\right]=\eta_{i j}=X_{i j}^{T} \gamma+Z_{i j}^{T} u_{j}
$$

for level-1 unit $i$ nested within level-2 unit $j$. At level 1, we assume $Y_{i j}$ conditionally distributed as Bernoulli, while the random effects vector $u_{j}$ is distributed as $N\left(0, \sigma_{u}^{2}\right)$ across the level-2 units. Let us consider the variance $\sigma_{u}^{2}$ as $T$ throughout this PQL estimation procedure.

The PQL approach can be derived as a nonlinear regression model. In the case of binary outcomes with logit link, we start with the level-1 model

$$
Y_{i j}=p_{i j}+e_{i j}
$$

where $E\left(e_{i j}\right)=0$ and $\operatorname{Var}\left(e_{i j}\right)=p_{i j}\left(1-p_{i j}\right)$. This is a nonlinear model which we linearize by means of the first-order Taylor series expansion. At this iteration $s$, we have

$$
p_{i j} \approx p_{i j}^{(s)}+\frac{d p_{i j}}{d \eta_{i j}}\left(\eta_{i j}-\eta_{i j}^{(s)}\right)
$$


and evaluate the derivative

$$
\frac{d p_{i j}}{d \eta_{i j}}=p_{i j}\left(1-p_{i j}\right)=\omega_{i j}
$$

at $p_{i j}^{(s)}$. Substituting the linear approximation for $p_{i j}$ in equation (2.1) yields

$$
Y_{i j}=p_{i j}^{(s)}+\omega_{i j}^{(s)}\left(\eta_{i j}-\eta_{i j}^{(s)}\right)+e_{i j}
$$

Algebraically rearranging this equation so that all known quantities are on the left-hand side of the equation produces

$$
\frac{Y_{i j}-p_{i j}^{(s)}}{\omega_{i j}^{(s)}}+\eta_{i j}^{(s)}=\eta_{i j}+\frac{e_{i j}}{\omega_{i j}^{(s)}} .
$$

This equation has the form of the familiar two-level hierarchical linear model

$$
Y_{i j}^{*(s)}=X_{i j}^{T} \gamma+Z_{i j}^{T} u_{j}+\varepsilon_{i j},
$$

which gives a straightforward updating scheme. This is known as penalizied quasi-likelihood because it is obtained by optimizing a quasi-likelihood (involving only 1 st and 2 nd derivatives) with a penalty term on the random effects. Here, $Y_{i j}^{*(s)}=\left(Y_{i j}-p_{i j}^{(s)}\right) / \omega_{i j}^{(s)}+\eta_{i j}^{(s)}, \varepsilon_{i j}=e_{i j} / \omega_{i j}^{(s)} \sim N\left(0, \omega_{i j}^{(s)-1}\right)$ and $u_{j} \sim N(0, T)$.

The estimate of $\eta_{i j}^{(s)}$ can be written as below

$$
\eta_{i j}^{(s)}=X_{i j}^{T} \hat{\gamma}^{(s)}+Z_{i j}^{T} u_{j}^{*(s)}
$$

where $u_{j}^{*(s)}$ is the approximate posterior mode, i.e.

$$
u_{j}^{*(s)}=\left(Z_{j}^{T} W_{j}^{(s)} Z_{j}+T^{(s)-1}\right)^{-1} Z_{j}^{T} W_{j}^{(s)}\left(Y_{j}^{*(s)}-X_{j} \hat{\gamma}^{(s)}\right)
$$

for $W_{j}^{(s)}=\operatorname{diag}\left\{\omega_{i j}^{(s)}, \ldots ., \omega_{n_{j} j}^{(s)}\right\}$.

\section{Data and Variables}

The dataset used in this study has been taken from the Bangladesh Demographic and Health Survey (BDHS) conducted in 2004. The 2004 BDHS is a nationally representative survey of 11440 ever-married women aged 10-49 from 10500 households covering 361 sample points (clusters) throughout Bangladesh, 122 in urban areas and 239 in the rural areas. The survey utilized a multistage cluster sample based on the 2001 Bangladesh Census and was designed to obtain 
and provide information on the basic indicators of social progress like fertility, childhood mortality, reproductive and child health etc.

This multistage 2004 BDHS dataset is of hierarchical structure. The hierarchy for this study follows individuals as level-1, clusters as level-2 and divisions as level-3. The nested structure of the study data is described in Figure 1. The 2004 BDHS survey did not collect any information on currently using contraception for the ever-married women who are currently pregnant as well. Therefore in order to avoid selection bias we omit pregnant women from the analysis. Among the ever-married women 724 were found pregnant, which left the number of evermarried women 10716 as our study population. After excluding 12 further women with missing explanatory variable our final study population becomes 10704 . The target population is "ever-married non pregnant women aged 10-49".

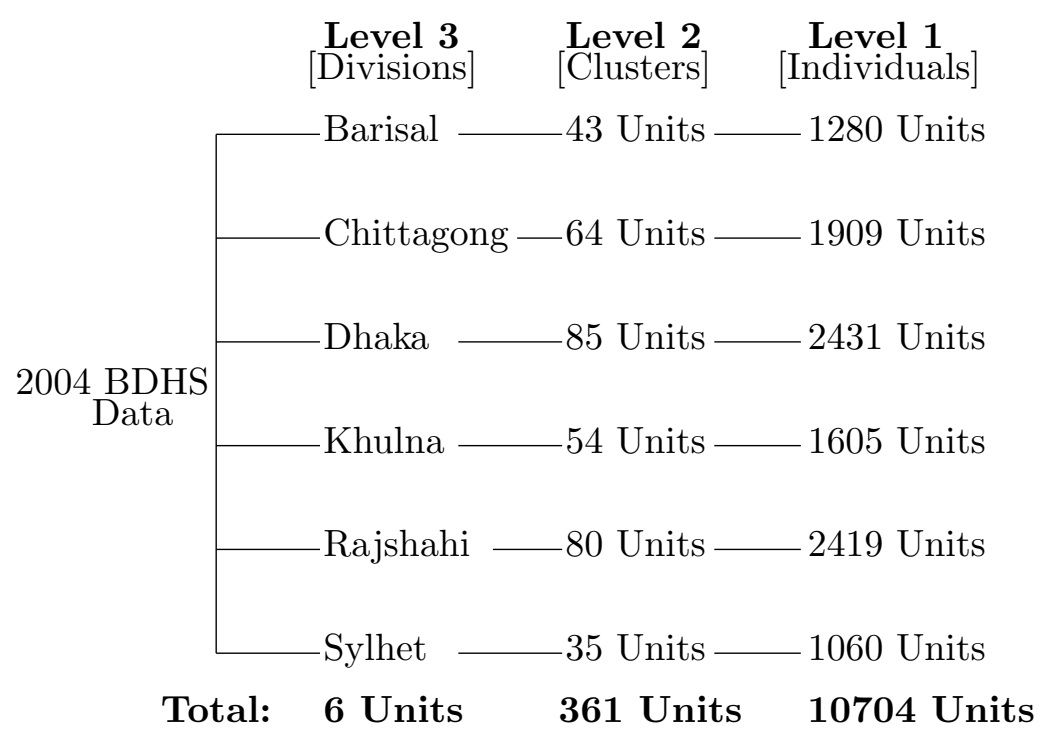

Figure 1: Hierarchical structure of the 2004 BDHS data

The dependent variable used for the analysis is currently using contraception (CUC). For the study purpose the response variable CUC is recoded as follows: those women currently using any of the methods - modern, traditional and folkloric - are coded as 1 and those not currently using any method are coded as 0 . The primary choice of explanatory variables for this study was based on previous other studies on the factors influencing contraceptive prevalence rate (Khan, 1997; Nashid et al., 1999; S. Amin et al., 2002; Kalam and Khan, 2002). 
Current age (CAge), number of living children (NOLC), education (Educ), religion (Reli), place of residence (POR), media and wealth index (WI) are the explanatory variables used in the study.

\section{Analysis and Results}

In order to use $M L w i N$ or a similar package for multilevel analysis, it is convenient to organize the data to reflect the data's hierarchical structure in the analysis. The BDHS data were therefore first sorted in such a way so that all records for the same highest level (level-3: Divisions) unit are grouped together and within this group, all records for a particular lower level (level-2: Clusters) unit are contiguous and within this group, all records for the lowest level (level1: Individuals) unit are also contiguous. All the selected covariates used in this study found significant in the bivariate analysis which was done before to start multilevel analysis. The multilevel process was stepwise. The first step examined the null model of overall probability of contraceptive use without adjustment for predictors. Second step included firs the univariate analysis (both single and multilevel), and then random slop multilevel univariate analysis for each of the selected explanatory variables. Third step considered a model building for threelevel multivariate logistic regression analysis including single level multivariate analysis. The Wald $\chi^{2}$ test was used to determine significance of each model as a whole as well as to determine significance of individual $\beta$ coefficients.

\subsection{Intercept Only Multilevel Logistic Model}

We first fit a simple model with no predictors i.e. an intercept-only model that predicts the probability of contraceptive use. The functional form of the model is

$$
\ln \left[\frac{p_{i j k}}{1-p_{i j k}}\right]=\beta_{0 j k}=\beta_{0}+v_{0 k}+u_{0 j k} .
$$

The estimates of parameters and standard errors are presented in Table 1. The ML estimate from the standard logit model of the ratio of contraceptive user to contraceptive nonuser is $\exp (0.299)=1.35$, which is the same as the sample ratio of 6146 contraceptive users to 4558 nonusers. It is in fact odds-ratio when no predictors have been considered in the model. In comparison, the same ratio is estimated to be $\exp (0.209)=1.23, \exp (0.227)=1.26, \exp (0.216)=1.24, \exp (0.221)=1.25$ and $\exp (0.333)=1.40$ from the multilevel model by the MQL-1, MQL-2, PQL-1, PQL-2 and MCMC methods respectively. The odds ratio from standard logit model can not be compared directly with odds ratios from a multilevel model since odds ratios from a multilevel model are effects on the median odds of using contraceptives whereas odds ratio estimated from a single-level has effect 
on the mean odds of using contraceptives. A crude (it is crude since each of mean and median is a measure of central tendency) comparison has been made to understand the multilevel effects. Compared to the odds-ratios obtained by all multilevel methods except MCMC the standard logistic model odds-ratio has overestimated. It is observed that there is a significant difference between the standard logistic estimate and the multilevel logistic estimate. Therefore, by failing to take into account the clustering within divisions (level 3) and clusters (level 2 ), the standard logistic model has overestimated the odds-ratio by about $43 \%$ $[(0.299-0.209) * 100 / 0.209], 32 \%, 38 \%$ and $33 \%$ compared to multilevel model using by the corresponding methods MQL-1, MQL-2, PQL-1 and PQL-2 (see Table 1). When the MCMC method has been applied the standard logistic model has underestimated the odds-ratio by about $11 \%$. The random quantity including its standard error at cluster level is similar to PQL-2 method but the division level random quantity is too high compared to all other methods.

Table 1: Parameters and standard errors of an intercept-only logit model and an intercept-only multilevel model predicting the probability of contraceptive use (S.E.s are placed in parentheses)

\begin{tabular}{|c|c|c|c|c|c|c|}
\hline \multirow[t]{2}{*}{ Model effect } & \multirow{2}{*}{$\begin{array}{c}\text { Standard logit } \\
\text { Logit }\end{array}$} & \multicolumn{5}{|c|}{ Multilevel models } \\
\hline & & MQL-1 & MQL-2 & PQL-1 & PQL-2 & MCMC \\
\hline Fixed effect & & & & & & \\
\hline Intercept & $\begin{array}{c}0.299 \\
(0.020)\end{array}$ & $\begin{array}{c}0.209 \\
(0.185)\end{array}$ & $\begin{array}{c}0.227 \\
(0.186)\end{array}$ & $\begin{array}{c}0.216 \\
(0.193)\end{array}$ & $\begin{array}{c}0.221 \\
(0.198)\end{array}$ & $\begin{array}{c}0.333 \\
(0.204)\end{array}$ \\
\hline Random effect & & & & & & \\
\hline Intercept(at level 2) & & $\begin{array}{c}0.123 \\
(0.020)\end{array}$ & $\begin{array}{c}0.124 \\
(0.020)\end{array}$ & $\begin{array}{c}0.149 \\
(0.022)\end{array}$ & $\begin{array}{c}0.156 \\
(0.023)\end{array}$ & $\begin{array}{c}0.158 \\
(0.025)\end{array}$ \\
\hline Random effect & & & & & & \\
\hline Intercept(at level 3) & & $\begin{array}{c}0.201 \\
(0.118)\end{array}$ & $\begin{array}{c}0.202 \\
(0.119)\end{array}$ & $\begin{array}{c}0.219 \\
(0.129)\end{array}$ & $\begin{array}{c}0.229 \\
(0.135)\end{array}$ & $\begin{array}{c}0.427 \\
(0.508)\end{array}$ \\
\hline$-2 \log \mathrm{L}$ & 14602.5 & & & & & \\
\hline Deviance & & & & & & 13799.1 \\
\hline $\mathrm{N}$ & 10704 & 10704 & 10704 & 10704 & 10704 & 10704 \\
\hline
\end{tabular}

Rodriguez and Goldman (1995) compared four approximation estimation procedures (first-order MQL or MQL-1, second-order MQL or MQL-2, first-order PQL or PQL-1, and second-order PQL or PQL-2) with the maximum likelihood achieved through high-dimensional numerical integration and the method of Gibbs sampling. They concluded that all approximation methods underestimate the random as well as fixed effects and that the underestimations of all except PQL-2 are severe. They preferred PQL-2 to all other methods as it has been found least biased. Since their research in 1995 it has been a norm to prefer the PQL-2 method as a multilevel estimation technique for binary data in many 
socio-economic and demographic studies when the estimates across other methods vary significantly (Goldstein, 2003). In the context of our analysis throughout the study we also preferred PQL-2 method to all other methods including MCMC.

The parameters under random effect in Table 1 are the estimated variances of the random intercepts at both levels (level 2 and 3) for fitting a three-level intercept-only model. In this three-level intercept-only model to understand the random effect, we can imagine a unique effect for each division (level 3) and for each cluster (level 2) in addition to the fixed intercept of 0.221 (PQL-2 estimate), which is the average of all divisions or all clusters. The addition of the cluster specific effects as well as division specific effects makes the model more accurate than the fixed intercept only model. In the random effect model, the cluster and division specific effects are assumed to be distributed normally for the purpose of estimation. In Table 1 the estimate of the random effect at both levels does increase as we go from MQL-1 to MQL-2, to PQL-1, to PQL-2, and even to MCMC. But the increases are much smaller than those reported by Rodriguez and Goldman (1997), and the increases between MQL-1 and MQL-2 and also between PQL-1 and PQL-2 are minuscule.

When the multilevel PQL-2 method is applied the expected log-odds of contraceptive use is 0.221 , corresponding to an odds of $\exp (0.221)=1.25$. This corresponds to a predicted probability of $1 /(1+\exp (-0.221))=0.555$. But for the standard logistic model the predicted probability is $1 /(1+\exp (-0.299))=0.574$. Assuming the division's log-odds of contraceptive use, $\beta_{0 j k}$, to be approximately normally distributed with mean 0.221 and variance, $\mathrm{V}\left(\beta_{0 j k}\right)=\mathrm{V}\left(\beta_{0}+v_{0 k}+\right.$ $\left.u_{0 j k}\right)=0.156+0.229=0.385$. The $95 \%$ confidence interval for $\beta_{0 j k}$ is $0.221 \pm$ $1.96 * \sqrt{0.385}=(-0.995,1.437)$. Converting these log-odds to predicted probabilities the $95 \%$ CI for predicted probability is $(0.25,0.81)$. That is, the contraceptive use rate or CPR varies from 25 percent to 81 within the divisions when multilevel effects have been considered and no predictor has been included in the model.

\subsection{Multilevel Univariate Logistic Mode}

In the multilevel univariate analysis represented in Table 2 each of the models presents a random intercept and a fixed slope for the variable. That is, it amounts to fitting a set of parallel straight lines to the predicted response currently using contraception (CUC) from the different units of a specific level. The 4th column of Table 2 represents odds ratios $(\hat{\psi})$ of the standard logistic model. Converting parameter estimates into odds ratios for multilevel logistic regression, as is done in standard logistic regression, is difficult, due to this model multilevel nature. In logistic regression, the odds of outcome for a non reference case in a predictor variable divided by the odds of outcome for a reference case for the same predictor variable does not depend on the other predictor variables. Thus, although odds 
Table 2: Parameters and standard errors of univariate single level logistic model and univariate multilevel model predicting the probability of contraceptive use with random intercept and fixed slope using PQL-2 method (S.E.s are placed in parentheses)

\begin{tabular}{|c|c|c|c|c|c|c|}
\hline \multirow[b]{2}{*}{ Row } & \multirow[b]{2}{*}{ Parameter } & \multicolumn{2}{|c|}{ Single level model } & \multicolumn{3}{|c|}{ Multilevel model } \\
\hline & & $\hat{\beta}_{s}$ & $\hat{\psi}$ & $\hat{\beta_{m}}$ & $\begin{array}{c}\text { Cluster } \\
\text { (Level-2) }\end{array}$ & $\begin{array}{l}\text { Division } \\
\text { (Level-3) }\end{array}$ \\
\hline 1 & CAge $^{1}$ & 0.000 & 1.000 & -0.002 & $0.156^{* *}$ & $0.229^{*}$ \\
\hline 2 & & $(0.001)$ & & $(0.002)$ & $(0.023)$ & $(0.136)$ \\
\hline 3 & $15-19$ & $0.469^{* *}$ & 1.598 & $0.575^{* *}$ & $0.151^{* *}$ & $0.252^{*}$ \\
\hline 4 & & $(0.203)$ & & $(0.209)$ & $(0.023)$ & $(0.148)$ \\
\hline 5 & $20-24$ & $0.824^{* *}$ & 2.280 & $0.984^{* *}$ & - & - \\
\hline 6 & & $(0.200)$ & & $(0.207)$ & $(-)$ & $(-)$ \\
\hline 7 & NOLC & & & & & \\
\hline 8 & 1 & $1.283^{* *}$ & 3.607 & $1.306^{* *}$ & $0.179^{* *}$ & $0.251^{*}$ \\
\hline 9 & & $(0.081)$ & & $(0.085)$ & $(0.025)$ & $(0.148)$ \\
\hline 10 & 2 & $1.667^{* *}$ & 5.296 & $1.708^{* *}$ & - & - \\
\hline 11 & & $(0.080)$ & & $(0.084)$ & $(-)$ & $(-)$ \\
\hline 12 & Educ & & & & & \\
\hline 13 & Primary & $0.039^{*}$ & 1.040 & $0.134^{* *}$ & $0.138^{* *}$ & $0.220^{*}$ \\
\hline 14 & & $(0.024)$ & & $(0.050)$ & $(0.021)$ & $(0.130)$ \\
\hline 15 & Secondary & $0.071^{* *}$ & 1.074 & $0.220^{* *}$ & - & - \\
\hline 16 & & $(0.025)$ & & $(0.054)$ & $(-)$ & $(-)$ \\
\hline 19 & & & & & & \\
\hline 20 & Reli & $-0.071^{* *}$ & 0.931 & $-0.421^{* *}$ & $0.138^{* *}$ & $0.246^{*}$ \\
\hline 21 & & $(0.031)$ & & $(0.078)$ & $(0.022)$ & $(0.145)$ \\
\hline 22 & & & & & & \\
\hline 23 & POR & $0.082^{* *}$ & 1.085 & $0.356^{* *}$ & $0.127^{* *}$ & $0.225^{*}$ \\
\hline 24 & & $(0.020)$ & & $(0.059)$ & $(0.021)$ & $(0.133)$ \\
\hline 25 & & & & & & \\
\hline 26 & Media & $0.058^{* *}$ & 1.058 & $0.219^{* *}$ & $0.139^{* *}$ & $0.231^{*}$ \\
\hline 27 & & $(0.019)$ & & $(0.042)$ & $(0.022)$ & $(0.136)$ \\
\hline 28 & WI & & & & & \\
\hline 29 & Middle & 0.034 & 1.035 & $0.141^{* *}$ & $0.127^{* *}$ & $0.239^{*}$ \\
\hline 30 & & $(0.028)$ & & $(0.059)$ & $(0.021)$ & $(0.141)$ \\
\hline 31 & Rich & $0.080^{* *}$ & 1.080 & $0.369^{* *}$ & - & - \\
\hline 32 & & $(0.022)$ & & $(0.050)$ & $(-)$ & $(-)$ \\
\hline
\end{tabular}

Note: The symbol $* *$ and $*$ indicate that the estimate is significant at 0.01 and 0.10 respectively. Reference categories are: '10-14' for CAge, '0' for NOLC, 'No education' for Educ, 'Non-Islam' for Reli, 'Rural' for POR, 'No TV or Radio' for Media, and 'Poor' for WI.

ratios can be calculated from the log odds, odds ratios cannot be isolated in the multilevel model in a comparable manner to logistic regression. To correctly interpret the parameter estimates related to predictors in a multilevel model, it 
is more meaningful to state that the individual estimates increase or decrease the log odds of the outcome. Another possibility is to convert the log odds into probabilities. We present $\beta$ coefficients (for notational convenience, $\beta_{s}$ for singlelevel and $\beta_{m}$ for multi-level; see in Table-2 and Table-3) for both type of models.

It is observed that there exist significant differences between the $\beta$ coefficients of these two models for each of the explanatory variables. Also the $\beta$ coefficients of the standard model have been underestimated in comparison with the multilevel model. The difference in $\beta$ coefficients estimated from a multilevel and standard model arises because of the addition of the random effects, imply that a singlelevel model for this outcome variable is not appropriate.

Results of first two rows (1 and 2) of Table 2 are related to current age of the women (CAge) when age is considered as a continuous variable measured in years. The results show that CAge is not significantly influencing CUC though there is a strong significant random effect at cluster and moderately significant (at 10\%) random effect at division level.

But when CAge is considered as a categorical variable (only two categories are shown in Table 2) the univariate model (from row 3 to 6 ) shows that the use of contraceptives largely depends on a woman's age category. Probably, it is due to the significant differences in contraceptive use between the age groups which is not so between the consecutive ages. For age group 15-19 the variance between intercepts of straight lines fitted for 6 divisions is 0.252 with standard error 0.148 . This reflects the existence of significant (at 10\%) differences between the mean effects due to this variable obtained from different divisions. This implies that some divisions are showing a higher tendency towards contraceptive use as age increases, and on the other hand some are showing a lower tendency. The estimates from Table 2 also reveal similar pictures for the case of other explanatory variables (for variables NOLC and Educ, results of only two categories are shown in Table 2). When these multilevel effects have not been taken into consideration, the $\beta$ coefficients have been underestimated for CAge. For instance, for age category $15-19$ and $20-24$ the $\beta$ coefficients of the single level model have been underestimated by almost $23 \%$ and $20 \%$ respectively. Similarly the $\beta$ coefficients for all the other explanatory variables except Reli have been underestimated. For Reli the $\beta$ coefficient has been overestimated when multilevel effects have not considered. Hence $\beta$ coefficients are distorted somewhat in both directions either in over or under direction from the true value when multilevel effects are not taken into consideration in modeling.

\subsection{Random slope multilevel univariate model}

Random effects univariate model allows the effect that the coefficient of the explanatory variable to vary from division to division and from cluster to cluster. 
We ran this model for each covariate separately and found only variable 'place of residence' (POR) has significant $(p<0.10)$ random effects across divisions. That is, the model allows the difference between urban and rural areas within a division to vary across divisions. Figure 2 shows that the predicted values vary across the six divisions between urban and rural and the predicted values for Chittagong and Sylhet division deviate the furthest from the line of equity. If we regard the clusters as a random sample from a population of clusters, then we wish to specify a coefficient of POR that is random at level 2. Similarly, considering division as a random effect will incorporate a coefficient of POR which is random at level 3.

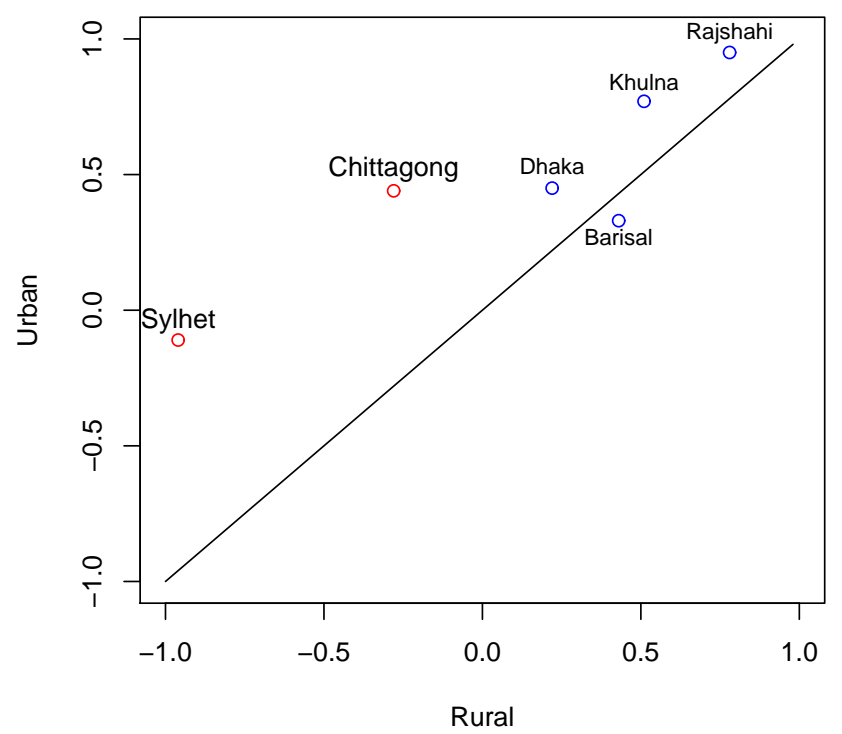

Figure 2: Division level (level-3) predicted points fitted by univariate model with random intercept and slope for variable 'Place of Residence' (POR).

The three-level random model for POR can be written as below.

$$
\ln \left[\frac{p_{i j k}}{1-p_{i j k}}\right]=\beta_{0}+\beta_{1} \mathrm{POR}_{i j k}+v_{0 k}+u_{0 j k}+\left(v_{1 k}+u_{1 j k}\right) \mathrm{POR}_{i j k},
$$

where the additive term $v_{0 k}+u_{0 j k}+\left(v_{1 k}+u_{1 j k}\right) \mathrm{POR}_{i j k}$ is in fact the residual $\left(E_{i j k}\right)$ of the model which is a function of POR. The variance of the model residual is always important in consideration of model adequacy, and is calculated from $\mathrm{V}\left(\mathrm{E}_{i j k}\right)=\mathrm{V}\left\{v_{0 k}+u_{0 j k}+\left(v_{1 k}+u_{1 j k}\right) \mathrm{POR}_{i j k}\right\}$; the estimates are found $\mathrm{V}_{\text {Rural }}\left(\mathrm{E}_{i j k}\right)=0.45$ and $\mathrm{V}_{\text {Urban }}\left(\mathrm{E}_{i j k}\right)=0.148$. That is, there is significantly greater 
division-level variation in the probability of using contraception in rural areas than in urban areas. This residual variation across the urban and rural region was considered fixed in the earlier univariate multilevel fixed effect model (Table 2 ). Therefore, to find out the actual effect of the parameters in modeling the random effect for POR has to be considered.

Table 3: Parameters and standard errors of single level multivariate logistic model and multilevel multivariate model predicting the probability of contraceptive use with random intercept, random slope for POR and fixed slope for others using PQL-2 method (S.E.s are placed in parentheses)

\begin{tabular}{|c|c|c|c|c|}
\hline \multirow[t]{2}{*}{ Parameter } & \multicolumn{2}{|c|}{ Single level model } & \multirow{2}{*}{$\begin{array}{l}\text { Multilevel model } \\
\hat{\beta_{m}}\end{array}$} & \multirow{2}{*}{$\begin{array}{c}\text { Over/Under } \\
\text { Estimation(\%) }\end{array}$} \\
\hline & $\hat{\beta}_{s}$ & $\hat{\psi}$ & & \\
\hline \multicolumn{5}{|l|}{ Fixed parameter } \\
\hline Intercept & $-1.722^{* *}(0.105)$ & 0.179 & $-1.907^{* *}(0.307)$ & 10.7 \\
\hline CAge & $-0.040^{* *}(0.003)$ & 0.961 & $-0.051^{* *}(0.003)$ & 27.5 \\
\hline \multicolumn{5}{|l|}{ NOLC } \\
\hline 1 & $1.432^{* *}(0.084)$ & 4.187 & $1.499^{* *}(0.086)$ & 4.7 \\
\hline 2 & $2.118^{* *}(0.087)$ & 8.314 & $2.250^{* *}(0.088)$ & 6.2 \\
\hline \multicolumn{5}{|l|}{ Educ } \\
\hline Primary & $0.142^{*}(0.051)$ & 1.153 & $0.119^{* *}(0.054)$ & 16.2 \\
\hline Secondary & $0.412^{* *}(0.059)$ & 1.510 & $0.370^{* *}(0.063)$ & 10.2 \\
\hline Reli & $-0.315^{* *}(0.067)$ & 0.730 & $-0.496^{* *}(0.079)$ & 57.5 \\
\hline POR & $0.312^{* *}(0.046)$ & 1.366 & $0.429^{* *}(0.150)$ & 37.5 \\
\hline Media & $0.164^{* *}(0.044)$ & 1.178 & $0.211^{* *}(0.047)$ & 28.7 \\
\hline \multicolumn{5}{|l|}{ Random parameter } \\
\hline$\sigma_{u 0}^{2}($ Intercept $)$ & & & $0.123^{* *}(0.026)$ & \\
\hline$\sigma_{u 5}^{2}(\mathrm{POR})$ & & & $0.001(0.001)$ & \\
\hline$\sigma_{u 05}$ & & & $-0.006(0.022)$ & \\
\hline$\sigma_{v 0}^{2}($ Intercept $)$ & & & $0.481^{* *}(0.195)$ & \\
\hline$\sigma_{v 5}^{2}(P O R)$ & & & $0.117^{* *}(0.059)$ & \\
\hline$\sigma_{v 05}$ & & & $-0.248^{* *}(0.107)$ & \\
\hline
\end{tabular}

Note: The symbol $* *$ and $*$ indicate that the estimate is significant at 0.01 and 0.05 respectively. Reference categories are: ' 0 ' for NOLC, 'No education' for Educ, 'Non-Islam' for Reli, 'Rural' for POR, and 'No TV or Radio' for Media.

\subsection{Multilevel multivariate logistic model}

The multivariate logistic model is followed with all the significant factors, found in the previous univariate analysis, to assess their simultaneous affect on contraceptive use. For multivariate analysis current age (CAge) is used as a 
continuous predictor rather than a categorical as is done in univariate analysis to avoid incorporating more variables in the model. Although the explanatory variable wealth index (WI) had played a significant role in univariate modeling, it is not seen as statistically significant in the multivariate analysis, probably due to the presence of multi-collinearity with some other model variables like education, media. The functional form of the multilevel model is:

$$
\begin{aligned}
\ln \left[\frac{p_{i j k}}{1-p_{i j k}}\right]= & \beta_{0 j k}+\beta_{1} \mathrm{CAge}_{i j k}+\beta_{2} \mathrm{NOLC}_{i j k}+\beta_{3} \mathrm{Educ}_{i j k}+\beta_{4} \operatorname{Reli}_{i j k} \\
& +\beta_{5 j k} \mathrm{POR}_{i j k}+\beta_{6} \text { Media }_{i j k}
\end{aligned}
$$

where $\beta_{0 j k}=\beta_{0}+v_{0 k}+u_{0 j k}$ and $\beta_{5 j k}=\beta_{5}+v_{5 k}+u_{5 j k}$. In fitting of this model we also went through data exploration or diagnostics techniques at both cluster and division level which suggest that two clusters (out of 361) had unusual residuals, leverages and influences and could be treated as extreme outliers. We finally fit the model (4.1) omitting these two clusters. Table 3 presents parameters ( $\beta_{s}$ for single-level and $\beta_{m}$ for multi-level model), standard errors and odds ratios from a single level logistic model predicting the probability of contraceptive use and its equivalent (except odds ratios) multilevel model. The last column of this table represents percentage of under or over estimation of $\beta$ coefficients by single level multilevel modeling.

The multivariate model shows that the probability of using contraception decreases significantly with age, adjusting for the effect of other predictors. When all other predictors are fixed in single-level multivariate analysis the probability of contraceptive use decreases $4 \%$ as age increases 1 year but for multilevel analysis the odds ratio can not be calculated directly. To compare multilevel and singlelevel analysis we compare their corresponding parameter estimates. From the last column of Table-3 it is seen that the $\beta$ coefficient under single-level analysis corresponding to age covariate has been overestimated about $27.5 \%$ compared to multilevel estimates.

As in the univariate analysis, number of living children (NOLC; only two categories are shown in Table-3) was found to be another important determinant to consider while predicting whether a woman will practice contraception. The $\beta$ coefficient for POR and Media from the standard logistic model have been greatly underestimated. On the other hand, the $\beta$ coefficient for Reli under standard logistic model has been greatly overestimated. Either notable overestimation or underestimation has happened for NOLC and Educ explanatory variables. In the multivariate framework variables, Educ (only two categories are shown in Table3), Reli, POR and Media have been found significantly associated with women's contraceptive use. The multilevel multivariate model has also revealed that there exist variations in the mean effect of the predictors (except for POR) over the 
response variable CUC in Bangladesh. The variation is significant $(p<0.01)$ at all levels of the hierarchy (lower, middle, and higher). In addition to the fixed effect the intercept has very strong significant random effects in cluster and division level. Also in addition to the fixed effect the only variable 'POR' has significant random effect at division level but not at cluster level. This indicates that variation among the mean effects obtained from 359 clusters and 6 divisions due to the linear combination of the selected model variables is significant. The random effect multilevel multivariate results imply that all the predictors are not equally and effectively defining the characteristics of women of all the clusters and all the divisions. Even within the place of residence (in urban or rural) the variation differ significantly across the division. That is, contraceptive use is correlated among women in the same place of residence within each division but the correlation differs from division to division.

\section{Discussion}

Very few multilevel analyses have been done in Bangladesh using contraceptive binary data, and these analyses have found significant multilevel effects either at lower levels (clusters, households, blocks) or middle level (districts) but not at higher level (such as division). For instance, S. Amin et al. (1996) found significant variation in the use of contraception at cluster and district level whereas Khan (1997), in his three level analysis, found significant variation in contraceptive use among lower levels (blocks) but not between higher levels (divisions). Kalam and Khan (2002) analysed 1996-97 BDHS contraceptive data and found significant variation in contraceptive use at lower levels (clusters, districts) but not at higher level (division). But our analysis reveals evidence $(p<0.1)$ of effects in higher level (divisions) in addition to higher significance in the lower level (clusters).

Our study has further demonstrated the tendency for the standard logistic model to seriously bias the parameter estimates of observed covariates when analyzing multilevel data. However, the estimated bias generally differs depending on the estimation procedure used for the multilevel logistic model. The differences between PQL-1 and PQL-2 as well as between MQL-1 and MQL-2 are minimal (Table-1). This is consistent with the observation in Goldstein and Rasbash (1996) that in the more common case where variances in a multilevel logistic model do not exceed about 0.5, the first order PQL model can be expected to perform well. That is, MLwiN software's PQL-1 and PQL-2 are likely to be adequate for producing nearly unbiased estimates. Further work can be done to investigate more precisely the relationship between the extent of bias and factors such as level of within-cluster correlation, level of within-division correlation, proportion of clusters that has a single observation, average size of clusters, number 
of clusters.

The 2004 BDHS contraceptive binary data is based on multistage stratified cluster sampling. Our study found that for such hierarchical structured data the multilevel effects are significant and have to be taken into consideration in logistic regression modeling, which leads to multilevel logistic regression modeling. As a result, this multilevel analysis enables the proper investigation of the effects of all explanatory variables measured at different levels (clusters and divisions) on the response variable 'currently using contraception', and finally the model produces appropriate estimates and conclusions about the parameters. A major reason for significant multilevel effects for such data might be dependencies between individual observations, due to sampling not being taken randomly but cluster sampling from geographical areas being used instead.

The univariate multilevel analysis (Table-2) of this study revealed that the mean effect of each of the predictors over current contraceptive use, viz., current age of the respondent (categorical), number of living children, education, religion, either having Radio or TV, wealth index varied significantly $(p<0.01)$ at cluster level and less significantly $(p<0.1)$ at division level. Also the predictor 'place of residence' has significant random effect in division level but not at cluster level. Similar results were found in multivariate multilevel analysis (Table-3). Mean effects of the combination of the predictors except wealth index varied significantly at cluster level $(p<0.01)$ and to a lesser extent $(p<0.1)$ at division level, and the random effect of place of residence varied considerably in division level. Thus multilevel analysis has demonstrated that different clusters and divisions have significantly different mean effects, and that the effect for place of residence is different in rural and urban areas across the divisions, a previously unrecognized effect.

\section{Acknowledgement}

The first author is grateful to the department of Statistics, University of Warwick, UK for the departmental bursary which had been offered for his MSc study.

\section{References}

Amin, R., Ahmed, A., Chowdhury, J., Kabir, M. and Hill, R. (1994). Recent evidence on trends and differentials in Bangladesh fertility: an update. Journal of Biosocial Science 26, 225-241.

Amin, S., Basu, A. M. and Stephenson, R. (2002). Spatial variation in contraceptive use in Bangladesh: Looking beyond the borders. Demography 39, 251-267. 
Amin, S., Diamond, I. and Steele, F. (1996). Contraception and religious practice in Bangladesh. In the continuing demographic transition. Research Working Paper No. 83, The Population Council, New York.

Breslow, N. E. and Clayton, D. G. (1993). Approximate inference in generalized linear mixed models. J. Am. Statist. Assoc. 88, 9-25.

Cleland, J., Phillips, J. F., Amin, S. and Kamal, G. M. (1994). The determinants of reproductive change in Bangladesh: success in a challenging environment (Tech. Rep.). World Bank Regional and Sectoral Studies.

Curtis, S. L. and Diamond, I. (1995). When fertility seems too high for contraceptive prevalence: An analysis of northeast Brazil. International Family Planning Perspectives 21, 58-63.

Goldstein, H. (1991). Nonlinear multilevel models with an application to discrete response data. Biometrika 78, 45-51.

Goldstein, H. (2003). Multilevel Statistical Models 3rd ed.. Oxford University Press.

Goldstein, H. and Rasbash, J. (1996). Improved approximations for multilevel models with binary responses. J. Roy. Statist. Soc. A 159, 505-513.

Islam, M. N. and Islam, M. M. (1993). Biological and behavioral determinants of fertility in Bangladesh : 1975-1989. Asia Pacific Population Journal 8, 3-18.

Kalam, E. M. N. and Khan, H. T. A. (2002). Modeling contraceptive prevalence in Bangladesh: A hierarchical approach. Asian Meta Centre, Research Paper Series, 21, N0. 5.

Khan, H. T. A. (1997). A hierarchical model of contraceptive use in urban and rural Bangladesh. Contraception 55, 91-96.

Laird, N. M. (1978). Empirical Bayes methods for two-way contingency tables. Biometrika 65, 581-590.

M. Anwarul Iqbal, B., Advisor. (2008). Country statement: Bangladesh (second draft). Paper presented at Special Governing Council Meeting of CRDAP and Ministerial Retreat, 24-26 June 2008, New Delhi, India.

Mauldin, W. P. and Segal, S. J. (1988). Prevalence of contraceptive use: trends and issues. Studies in Family Planning 19, 335-353.

Nashid, K., Sloggett, A. and Cleland, J. G. (1999). Are variations in the use of modern contraception in rural Bangladesh: A multilevel analysis. Journal of Biosocial Science 31, 327-341.

NIPORT. (1999-2000). Bangladesh Demographic and Health Survey 1999-2000 (Tech. Rep.). Calverton, Maryland: National Institute of Population Research and Training (NIPORT), Mitra and Associates, and ORC Macro, Dhaka, Bangladesh.

NIPORT. (2005). Bangladesh Demographic and Health Survey 2004 (Tech. Rep.). Calverton, Maryland: National Institute of Population Research and Training (NIPORT), Mitra and Associates, and ORC Macro, Dhaka, Bangladesh. 
Rani, S. U. and Radheshyam, B. (2007). Inconsistencies in the relationship between contraceptive use and fertility in Bangladesh. International Family Planning Perspectives 33, 31-37.

Rasbash, J., Steele, F., Browne, W. and Prosser, B. (2004). A user's guide to mlwin. Version 2, Centre for Multilevel Modelling, University of Bristol, UK.

Rodriguez, G. and Goldman, N. (1995). An assessment of estimation procedures for multilevel models with binary responses. Journal of the Royal Statistical Society, Series A 158, 73-89.

Rodriguez, G. and Goldman, N. (1997). Multilevel models with binary response: a comparison of estimation procedures. Paper Pres. Pop. Assoc. Am. Annu. Meet. Wasington, DC. March 27-29.

Received March 31, 2009; accepted April 30, 2009.

Md. Hasinur Rahaman Khan

Department of Statistics

University of Warwick

United Kingdom

hasinur@isrt.ac.bd

J. Ewart H. Shaw

Department of Statistics

University of Warwick

United Kingdom

Ewart.Shaw@warwick.ac.uk 